\title{
Nonlinear Dynamics of Long-Wavelength VCSELs subject to Polarised Optical Injection
}

\author{
A. Hurtado, K. Schires, R. Al-Seyab, N. Khan, I.D. Henning and M.J. Adams \\ School of Computer Science and Electronic Engineering, University of Essex \\ Wivenhoe Park, Colchester, CO4 3SQ, United Kingdom \\ ahurt@,essex.ac.uk
}

\begin{abstract}
We present our work analysing the effects of polarised optical injection in Long-Wavelength Vertical-Cavity Surface Emitting Lasers (LW-VCSELs). We review the properties of different phenomena such as polarisation switching (PS) and bistability (PB) as well as on the different nonlinear dynamics (periodic and chaotic) arising in these devices under different cases of polarised optical injection.
\end{abstract}

Keywords: Vertical-Cavity Surface Emitting Lasers (VCSELs), optical injection and nonlinear dynamics.

\section{INTRODUCTION}

Optically-injected LW-VCSELs show promise as low-cost sources for optical telecommunication networks [1]. Highly enhanced modulation bandwidth $(>100 \mathrm{GHz})$ has been demonstrated in injection locked 1550nm-VCSELs [2]. However, the effect of different polarised optical injection onto these devices had not received considerable research effort. Therefore in recent years we have focused onto the experimental and theoretical analysis of these effects reporting first on the properties of the Polarisation Switching (PS) and Bistability (PB) [3][4] induced in these devices when subject to orthogonally-polarised optical injection. Later we extended our studies to analyse the injection locking bandwidth and the different regions of nonlinear dynamics appearing when the devices were subject to different polarised optical injection [59]. We have reported experimental and numerical stability maps (in the plane of injection strength versus frequency detuning) determining the boundaries of regions of different dynamics [5-7]. Additionally, we have analysed in depth the characteristics of the dynamics under different regimes of polarised injection including the report of experimental timeand polarisation-resolved analyses [8][9]. The rich variety of nonlinear effects observed in LW-VCSELs emitting at the important telecom wavelength of $1550 \mathrm{~nm}$ offers exciting prospects for novel practical use of these devices in future optical networks.

\section{EXPERIMENTAL SETUP}

Fig. 1 shows the experimental arrangements [3-9]. Fig. 1(a) shows the part of the setup designed to controllably inject polarised light from a tuneable laser source into a $1550 \mathrm{~nm}$ VCSEL. For the analysis two different configurations were used. The first, shown in Fig. 1(b), was dedicated to study the properties of the total signal at the VCSEL's reflective output. This was analysed optically with an Optical Spectrum Analyser (OSA), and electrically using an Electrical Spectrum Analyser
(ESA) and a fast real-time oscilloscope. Fig. 1(c) shows the second configuration where the two individual polarised outputs from the VCSEL are separated using a Polarisation Beam Splitter (PBS) and are individually and simultaneously analysed using the fast real-time oscilloscope. Commerciallyavailable quantum-well (QW) 1550nm-VCSELs from the same manufacturer and showing very similar characteristics were used in this work [3-9]. Fig. 1(d) depicts the optical spectrum of the solitary device showing two modes (separated approx. $0.5 \mathrm{~nm}$ ) corresponding to the two orthogonal polarisations of the fundamental transverse mode of the device. Throughout this work we refer to parallel and orthogonal to the polarisation of the lasing and the subsidiary attenuated mode of the device.

\section{RESULTS}

Fig. 2 illustrates PS and PB obtained with a 1550nmVCSEL subject to orthogonally-polarised optical injection [3][4]. Figs. 2 (a) and (b) plot respectively the output optical power of the orthogonal and the parallel polarisation outputs of the VCSEL versus the optical input power. As seen in fig. 2, PS is obtained when a particular level in the input power is exceeded; bistability (PB) may also occur in association with PS for high enough levels of detuning. The small insets in the right part of fig. 2 plot the spectrum of the device before and after the injection of an orthogonally-polarised optical signal. The solitary device emits parallel polarised light; under external light injection, the orthogonal mode locks to the injected signal, the parallel mode is suppressed and the polarisation at the VCSEL's output switches from parallel to orthogonal. Always a similar nonlinear transition switching from a higher to a lower state (with or without bistability) is attained for the output of the parallel polarisation (fig. 2(b)) whereas a variety of switching transitions with different shapes, anticlockise or clockwise, (fig. 2(a)) appears in the output of the orthogonal polarization [3].

We have also studied experimentally and in theory the injection locking (IL) properties of these devices under different polarised optical injection [5-7]. As an example, fig. 3(a) shows the experimentally measured stability map (in the plane of injected power vs. frequency detuning) for a $1550 \mathrm{~nm}$ VCSEL subject to orthogonally polarised injection [7]. As seen in fig. 3(a) stable IL is only found in a small region around the VCSEL's resonant wavelength. Outside this region a rich variety of nonlinear dynamics are obtained [6-7]. These include limit cycle of period 1 (P1) dynamics with (P1\&PS, in green) or without (P1, in light blue) associated PS, Period doubling of 


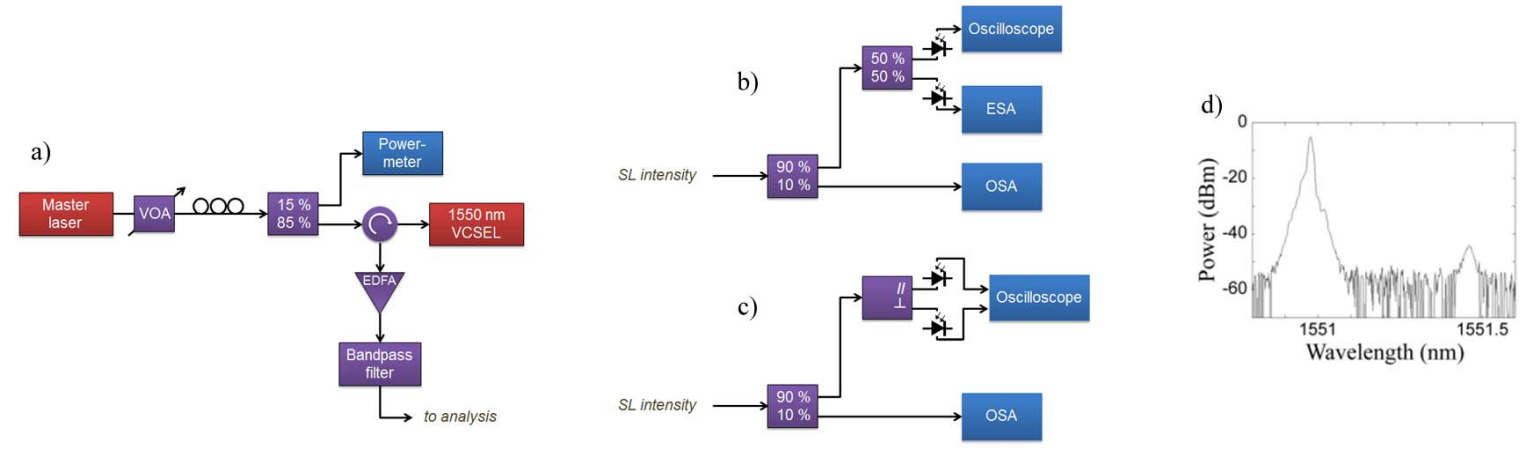

Fig. 1. Experimental setup: (a) Part of the setup dedicated to the external injection of an optical signal into the 1550nm-VCSEL. Two different configurations are used for the analysis part of the setup: (b) analysis of the total signal at the VCSEL's output and (c) individual study of both orthogonal polarisations outputs of the VCSEL. (d) Optical Spectrum of a 1550nm-VCSEL used in the experiments. [8,9]

(a)

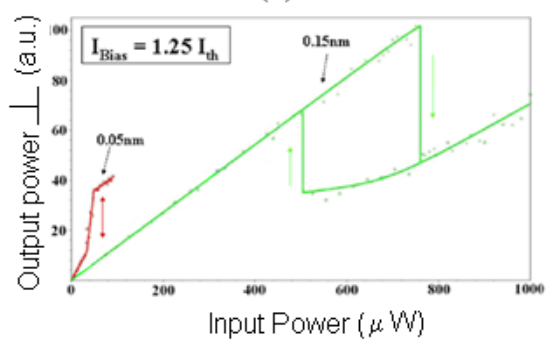

(b)

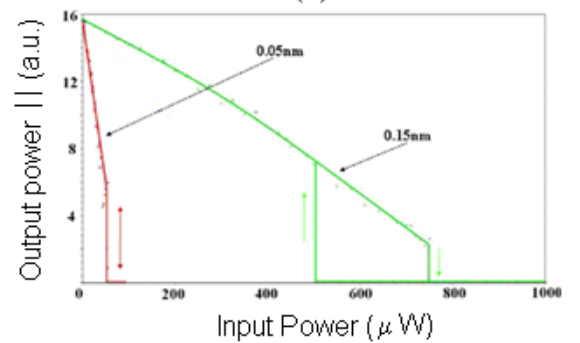

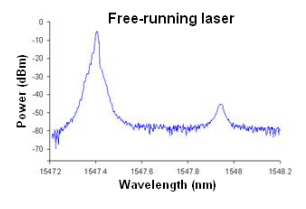

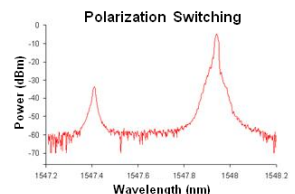

Fig. 2. PS and PB in a 1550nm-VCSEL. I/O power relationships for the orthogonal (a) and parallel (b) polarisation outputs of the VCSEL. The device is biased with a current equal to 1.25 times the value at threshold $\left(I_{\text {Bias }}=1.25 I_{t h}\right)$. Two different cases of detuning $(0.05$ and $0.15 \mathrm{~nm})$ are studied. The insets at the right part of the figure depict the optical spectrum of the VCSEL before and after the attainment of PS.
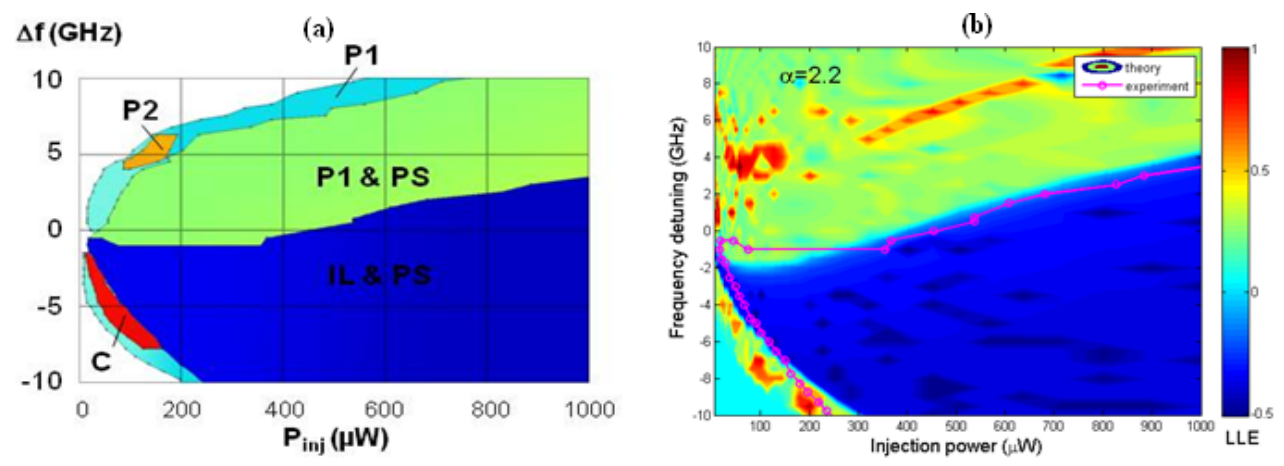

Fig. 3. Measured (a) and calculated (b) stability maps for the $1550 \mathrm{~nm}-\mathrm{VCSEL}$ subject to orthogonally polarised optical injection. (I=3I $\mathrm{t}_{\mathrm{th}}$ ). [7]

Period 2 (P2) (in orange) and chaotic dynamics (C, in red). We have analysed the system theoretically and Fig. 3(b) shows a numerically calculated stability map for the same conditions used in fig. 3(a). For this we have developed a novel extension of the Spin Flip Model including noise to simulate the operation of a VCSEL under different polarised optical injection. This SFM extension has been subsequently combined with the Largest Lyapunov Exponent method for the generation of numerically calculated stability maps. For complete details on the model, computation mechanisms and parameter values see [7]. The same colour code was used in figs. 3(a) and 3(b) to represent the different regions of nonlinear dynamics appearing outside the locking range. Overall, very good agreement is found between theory and experiments.

In addition to reporting experimental and numerical stability maps, we have also analysed the temporal characteristics of the different nonlinear dynamics for different cases of polarised injection. Initially, combining the experimental setup included in fig. 1(a) and the analysis part depicted in fig. 1(b) we measured simultaneously the optical and electrical spectra as well as the real-time series for the total signal at the VCSEL's reflective output. Phase plots were constructed plotting the signal intensity $I$ against its time derivative. Fig. 4 shows the case where different dynamics are obtained with a device subject to parallel polarised optical injection. The initial frequency detuning was held constant at $\Delta f=5 \mathrm{GHz}$ and the injection strength was varied from -12 to $1.3 \mathrm{~dB}$ [8]. First, fig. 4(c) shows the appearance of limit cycle dynamics where the VCSEL's output oscillates periodically at a given frequency (approx. $9 \mathrm{GHz}$ ) as it can be seen in the measured time series. The main peak appearing in the electrical spectrum and the side-mode separation in the optical spectrum 

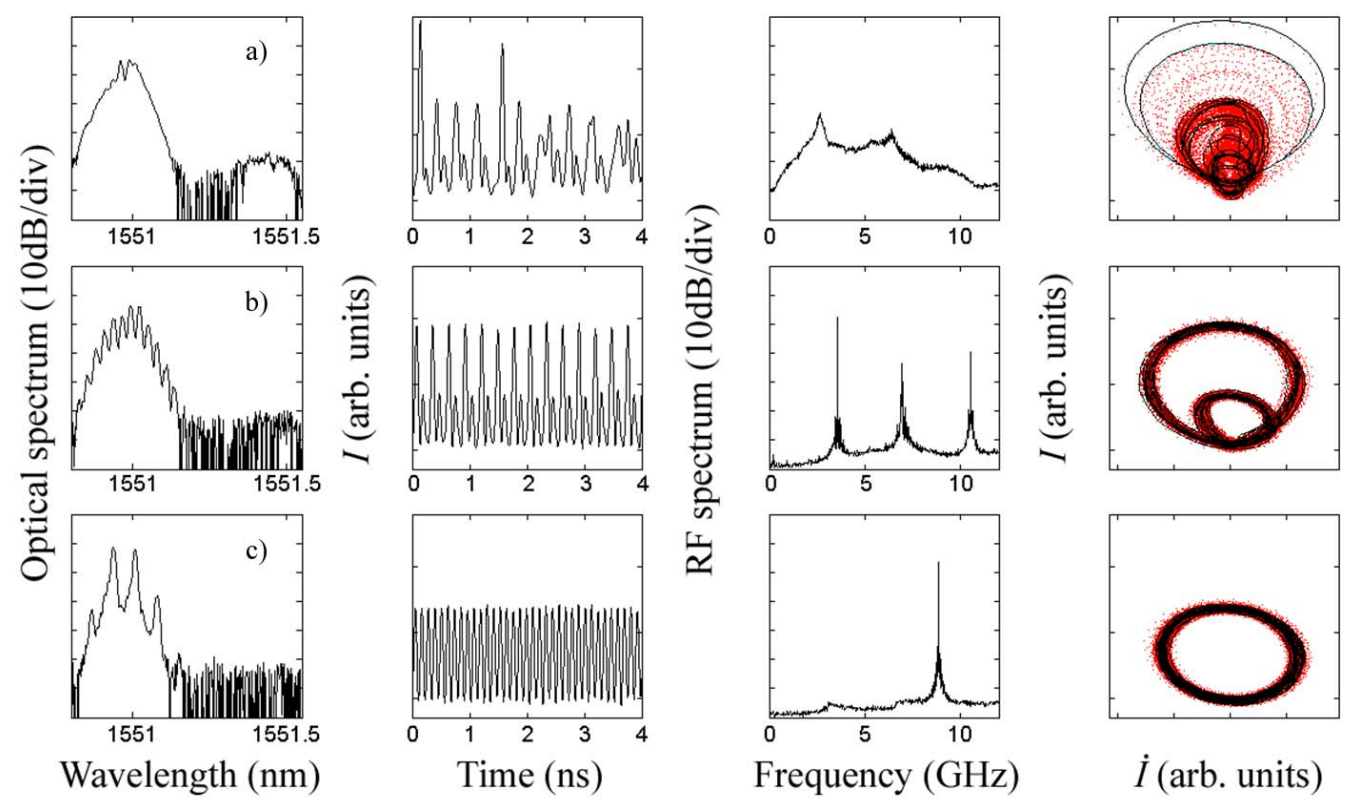

$\dot{I}$ (arb. units)

Fig. 4. From left to right: optical spectrum, real-time series, electrical spectrum and phase plots for the total signal at the VCSEL's output. The device is subject to parallel polarised optical injection with initial frequency detuning of $\Delta \mathrm{f}=5 \mathrm{GHz}$ and injection strength equal to (a) $-12 \mathrm{~dB}$, (b) $-2 \mathrm{~dB}$ and (c) $1.3 \mathrm{~dB}$. The black phase plot corresponds to the $4 \mathrm{~ns}$ time series displayed whereas the red phase plot is generated from $100 \mathrm{~ns}$ of recorded time series. [8]
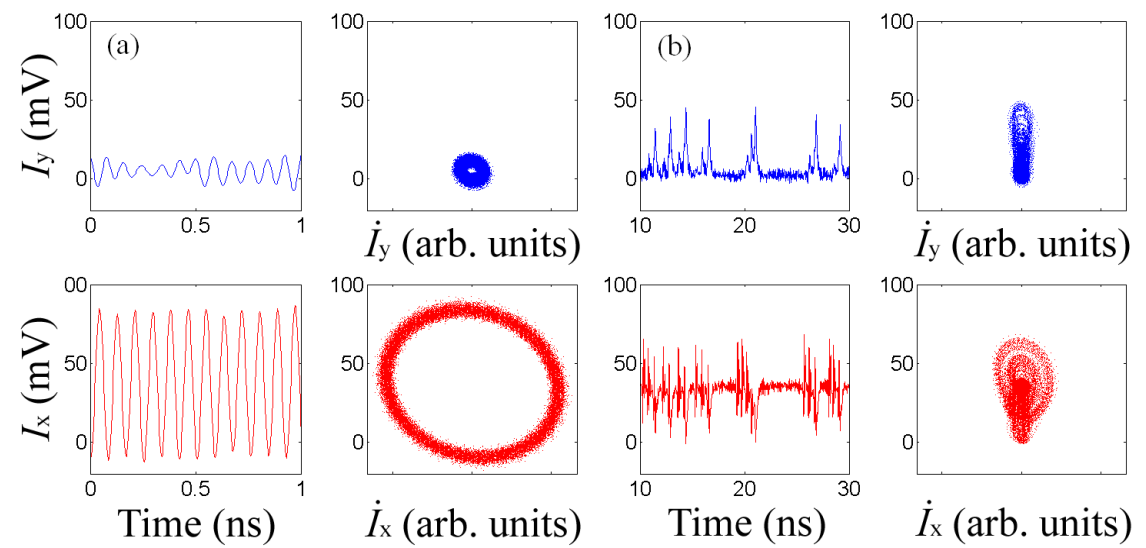

Fig. 5. Time series and phase plots of the parallel $\left(\mathrm{I}_{\mathrm{y}}\right.$, blue) and orthogonal $\left(\mathrm{I}_{\mathrm{x}}\right.$, red) polarisations at the VCSEL's output. The injection power and frequency detuning are respectively equal to: (a) $-1.1 \mathrm{dBm},-13.1 \mathrm{GHz}$ and (b) $-14.4 \mathrm{dBm},-0.9 \mathrm{GHz}$. [9]

corresponds with precisely the oscillation frequency. Finally, the phase plot shows a characteristic open circle shape where the radius of the circle is indicative of the amplitude of the oscillations. Fig. 4(b) shows a different situation, now period doubling dynamics appear at the VCSEL's output and in this case, the device's output oscillates periodically at two different frequencies being one the half of the other. The peaks at approx. 4 and $8 \mathrm{GHz}$ in the electrical spectrum indicate the magnitude of these two oscillation frequencies and the phase plot shows a characteristic double open circle shape. Finally, fig. 4(a) shows a situation where chaotic dynamics appear at the VCSEL's output. In this case the time series show an extremely non-periodical pattern, widen spectra appear in both the optical and electrical spectra and a very irregular shape characterises the phase plot.
We have also analysed the polarisation-resolved dynamics of a 1550nm-VCSEL under orthogonally-polarised optical injection [9]. For this purpose we combined the analysis part of the setup in fig. 1(c) with the main setup of fig. 1(a). With this configuration we were able to record simultaneously realtime series of the intensities of the two individual polarisations at the VCSEL's output. Fig. 5 shows time series and phase plots for the two polarisations, orthogonal $\left(I_{\mathrm{x}}\right.$, in red) and parallel $\left(I_{\mathrm{y}}\right.$, in blue), and for two different values of injected power and frequency detuning as indicated in the caption. Different types of nonlinear dynamics were simultaneously found in both polarisations. Specifically, fig. 5(a) shows the simultaneous occurrence of period 1 dynamics in both polarisations. In contrast, fig. 5(b) illustrates a completely different scenario where chaotic dynamics with similar amplitudes are obtained simultaneously in both polarisations. 


\section{CONCLUSIONS}

In this work we have reviewed our recent studies analysing the rich variety of nonlinear effects arising in LW-VCSELs under different polarised optical injection. We have described the PS and PB occurring in these devices under orthogonallypolarised optical injection. Additionally, we have also reviewed our studies on the diverse nonlinear dynamics appearing outside the locking range when a LW-VCSEL is subject to different polarised optical injection. We have reported experimental and numerical stability maps delimiting the boundaries between regions of different nonlinear dynamics, showing overall very good agreement between theory and experiments. We have also reported a complete description of the characteristics of the nonlinear dynamics using measured optical and electrical spectra and recorded time series, and have extended this to resolve simultaneously the two individual polarisations at the VCSEL's output. The rich variety of behaviours reported in this work for VCSELs operating at $1550 \mathrm{~nm}$ offers exciting prospects for novel practical uses of VCSELs in different fields spanning from optical signal processing to encrypted communication systems.

\section{ACKNOWLEDGMENT}

This work was supported in part by the European Commission under the Programme FP7 Marie Curie IntraEuropean Fellowships Grant PIEF-GA-2008-219682 and the U.K. Engineering and Physical Sciences Research Council (EPSRC) under grant reference EP/G012458/1.

\section{REFERENCES}

[1] C.-H. Hang, L. Chrostowski and C.J. Chang-Hasnain, "Injection locking of VCSELs," IEEE J. Select Topics Quantum Electron., vol. 9, pp. 1386-1393 (2003)

[2] E.K. Lau, X.X. Zhao, H.K. Sung, D. Parekh, C. Chang-Hasnain and M.C. Wu, "Strong optical injection-locked semiconductor lasers demonstrating $>100-\mathrm{GHz}$ resonance frequencies and $80-\mathrm{GHz}$ intrinsic bandwidths," Opt. Express, vol. 16, pp. 6609 (2008)

[3] A. Hurtado, I.D. Henning and M.J. Adams, "Two-wavelength switching with a $1550 \mathrm{~nm}$ VCSEL under single orthogonal optical injection," IEEE J. Select Topics Quantum Electron., vol. 14, pp. 911-917 (2008)

[4] A. Hurtado, A. Quirce, A. Valle, L. Pesquera, M.J. Adams, "Power and wavelength polarization bistability with very wide hysteresis cycles in a $1550 \mathrm{~nm}$-VCSEL subject to orthogonal optical injection," Opt. Express, vol. 17, pp. 23637-23642 (2009)

[5] A. Hurtado, D. Labukhin, I.D. Henning and M.J. Admas, "Injection locking bandwidth in $1550 \mathrm{~nm}$-VCSELs subject to parallel and orthogonal optical injection," IEEE J. Select Topics Quantum Electron., vol. 15, pp. 585-593 (2009)

[6] A. Hurtado, A. Quirce, A. Valle, L. Pesquera and M.J. Adams, "Nonlinear dynamics induced by parallel and orthogonal optical injection in $1550 \mathrm{~nm}$ vertical-cavity surface-emitting lasers," Opt. Express, vol. 18, pp. 9423-9428 (2010).

[7] R. Al-Seyab, K. Schires, N. Khan, A. Hurtado, I.D. Henning and M.J. Adams," "Dynamics of Polarised Optical Injection in 1550nm-VCSELs: Theory and Experiments," IEEE J. Select Topics Quantum Electron., vol. 17, pp. 1242-1249 (2011)

[8] K. Schires, A. Hurtado, I.D. Henning and M.J. Adams, "Comprehensive experimental analysis of nonlinear dynamics in an optically-injected semiconductor laser", AIP Advances, vol. 1, 032131 (2011)

[9] K. Schires, A. Hurtado, I.D. Henning and M.J. Adams, "Polarisation and time-resolved dynamics of a 1550nm VCSEL subject to orthogonallypolarised optical injection," IEEE Photonics Journal, vol. 3, pp. 555-563 (2011) 